\title{
Decreased amylolytic microbes of the hindgut and increased blood glucose implied improved starch utilization in the small intestine by feeding rumen-protected leucine in dairy calves
}

\author{
Hao Ren, () Hanxun Bai, Xiaodong Su, Jie Pang, Xiaoyong Li, Shengru Wu, ㄱ Yangchun Cao, 나 \\ Chuanjiang Cai, and Junhu Yao* () \\ College of Animal Science and Technology, Northwest A\&F University, Yangling, Shaanxi 712100, P.R. China
}

\begin{abstract}
Starch digestion in the small intestine in ruminants is relatively lower compared with that in monogastric animals, likely due to low pancreatic $\alpha$-amylase secretion. Previous studies suggested that leucine could increase pancreatic $\alpha$-amylase secretion in the small intestine of heifers cannulated with abomasal, duodenal, and ileal catheters. However, the surgical procedures probably have an effect on pancreatic function. Thus, we used rumen-protected leucine (RP-Leu) to explore its effect on small intestinal digestion of starch in calves without any surgery in 3 experiments. The first experiment was to explore whether RP-Leu could improve post-ruminal starch digestion in 5-mo-old calves $(158 \pm 19 \mathrm{~kg}$ body weight \pm standard deviation). We found that RP-Leu did not affect rumen fermentation profile or wholetract starch digestibility, but it increased blood glucose concentration and fecal $\mathrm{pH}$ and decreased fecal propionate molar proportion. Additionally, RP-Leu increased fibrolytic genera Ruminiclostridium and Pseudobutyrivibrio and decreased the amylolytic genus of Faecalibacterium. The second experiment compared RP-Leu and rumen-protected lysine (RP-Lys) for their effects on post-ruminal starch digestion in 6-mo-old calves (201 $\pm 24 \mathrm{~kg}$ body weight). The responses of blood glucose concentration, fecal $\mathrm{pH}$, fecal propionate proportion, and starch digestibility to RP-Leu supplementation were similar to those observed in experiment 1 . Cellulolytic family Ruminococcaceae and Bacteroidales BS11 gut group tended to be increased by RP-Leu. In contrast, RP-Lys showed no significant influence on the above measurements. The third experiment determined the interaction between RP-Leu and rumen-escape starch (RES) on the small intestinal digestion of starch in 8-mo-old calves (289 $\pm 26 \mathrm{~kg}$ body weight). An interaction between RP-Leu and RES levels was observed in
\end{abstract}

Received June 30, 2019.

Accepted December 18, 2019.

*Corresponding author: yaojunhu2004@sohu.com fecal butyrate concentration and the relative abundance of family Bacteroidaceae, and genera Ruminococcaceae UCG-005 and Bacteroides. We found that RP-Leu tended to increase the abundance of fecal Firmicutes and decrease Spirochaetae. In conclusion, RP-Leu, but not RP-Lys, increased blood glucose concentration and decreased the amount of starch fermented in the hindgut in a RES dose-dependent manner, suggesting that RP-Leu might stimulate starch digestion in the small intestine.

Key words: leucine, starch, fecal microbiota, $16 \mathrm{~S}$ rRNA gene sequencing

\section{INTRODUCTION}

With the increasing global consumption of milk and meat products, high-energy diets, especially highstarch diets, are usually formulated for dairy cows and beef cattle to pursue high milk yields and fast growth rates. This feeding approach, however, often leads to the occurrence of rumen acidosis and an increase in the amount of starch flowing into the small intestine. Compared with digestion in the rumen, starch digestion in the small intestine can provide $42 \%$ more energy (Owens et al., 1986, 2016; Harmon, 2009); therefore, shifting starch digestion from the rumen to the small intestine can significantly improve feed conversion efficiency. Starch digestibility in the small intestine of ruminants is limited to no more than $60 \%$ (Giuberti et al., 2014; Moharrery et al., 2014). This low digestibility is possibly attributable to the capacity for glucose absorption, the inadequate time for sufficient digestion, and the enzyme working conditions, as well as the limited pancreatic $\alpha$-amylase secretion (Huntington, 1997; Swanson et al., 2002; Harmon et al., 2004). Nonetheless, such a low starch digestibility could waste feed resources and increase production costs (Anderson and Trapp, 2000). It is difficult to improve small intestinal starch digestion by supplying exogenous amylase to ruminants, due to the rumen microbiome. In addition, abomasal infusion of amylase also seems to fail to 
increase postruminal starch digestion in heifers when different amounts of corn starch are abomasally infused (Westreicher-Kristen et al., 2018; Robbers et al., 2019). The lack of effect could have been masked by insufficient activity of other hydrolyzing enzymes, limited capacity for glucose absorption, or reduced endogenous pancreatic amylase secretion by exogenous amylase or starch infusion. Therefore, suitable nutrients and an appropriate supplementary approach are needed to improve pancreatic exocrine function and starch digestion in the small intestine.

Casein has been widely used to increase pancreatic weight and total pancreatic $\alpha$-amylase secretion (Swanson et al., 2002, 2004; Lee et al., 2013). The contents of certain amino acids in casein may influence its effect on the secretion of pancreatic $\alpha$-amylase (Swanson et al., 2004; Liao et al., 2009). Leucine is one of the essential amino acids, as a substrate for protein synthesis, and is also considered a functional molecule that stimulates protein synthesis $(\mathrm{Wu}, 2010)$. Leucine can promote $\alpha$-amylase synthesis in pancreatic acinar cells as a signal molecule in dairy calves, particularly via the mammalian target of rapamycin (mTOR) signaling pathways in vivo (Guo et al., 2018). To test the hypothesis that the stimulation of global protein synthesis by leucine could also enhance the synthesis of pancreatic $\alpha$-amylase, we previously installed abomasal, duodenal, and ileal catheters in dairy heifers (Liu et al., 2015) and goats (Yu et al., 2014) to deliver leucine directly into the duodenum so that its regulatory roles in the secretion of pancreatic $\alpha$-amylase could be tested. In those reports, we found that increasing the intestinal leucine flux enhanced the synthesis and secretion of pancreatic $\alpha$-amylase and further increased starch digestion in the small intestine. However, the potential influences of the surgery on pancreatic functions could not be ruled out (Ternouth and Buttle, 1973; Tiscornia et al., 1977; Zabielski and Pierzynowski, 2001; Ansia et al., 2019). Therefore, the current experiment 1 was to evaluate rumen-protected leucine (RP-Leu) supplementation for its ability to possibly improve small intestinal starch digestion in heifers without the confounding factor of surgery. On the other hand, previous studies delivered only leucine to examine its effect of acting as a molecular signal on small intestinal starch digestion, which could not exclude the role of amino acids as the limited precursor for protein synthesis. Thus, experiment 2 was designed to further try to determine the role of leucine as a regulatory molecule in starch digestion; lysine was included as the control for the role of essential amino acids in global protein synthesis, which may also affect small intestinal digestion of starch. Additionally, previous studies on leucine focused mainly on its regulatory roles in the secretion of pancreatic $\alpha$-amylase without considering the starch level flowing to the small intestine. Consequently, experiment 3 was designed to examine the interaction between RP-Leu and rumen-escape starch (RES) levels in small intestinal digestion of starch. Blood glucose concentration, whole-tract starch digestibility, fecal VFA profile, and fecal microbiota were analyzed as proxies of small intestinal starch digestion and metabolism.

\section{MATERIALS AND METHODS}

\section{Ethics Approval Statement}

This study was conducted in accordance with the guidelines of the Administration of Affairs Concerning Experimental Animals (Ministry of Science and Technology, Beijing, China, revised in 2004). The protocol was approved by the Institutional Animal Care and Use Committee of the Northwest A\&F University (protocol number: NWAFAC1211; Yangling, Shaanxi, China).

\section{Animals, Experimental Design, and Diets}

Experiment 1. Fourteen male Holstein calves (158 $\pm 19 \mathrm{~kg}$ of $\mathrm{BW}, \pm \mathrm{SD}$ ) were randomly assigned to 2 groups of 7 calves per group. The calves in the control group received the basal diet only, whereas calves in the experimental group were fed the basal diet supplemented with $36 \mathrm{~g} / \mathrm{d}$ of RP-Leu. The RP-Leu product (Hangzhou King Techina Technology Co. Ltd., Hangzhou, China) contained $70 \%$ leucine within a palm oil coating, which could provide an $80 \%$ rumen bypass rate in situ and a $98 \%$ abomasal release rate (Rossi et al., 2003). The dosage of RP-Leu was determined based on the results of previous studies (Rulquin and Pisulewski, 2006; Liu et al., 2015).

The experiment lasted $28 \mathrm{~d}$, with the first $21 \mathrm{~d}$ for adaptation and the remaining $7 \mathrm{~d}$ for sampling. The diet was formulated to meet the nutrient requirements for dairy calves (about $1.5 \times$ maintenance energy requirement, NRC, 2001). The ingredients and nutritional composition of the diet are shown in Table 1. The diet was formulated to provide adequate ruminally available $\mathrm{N}$ and to meet or exceed the requirements for vitamins and minerals, and it was also designed to provide only modest amounts of RUP so that the MP supply would be moderate. Calves were housed in individual pens $(7 \mathrm{~m} \times 4 \mathrm{~m})$ in a barn with sawdust bedding and a feed lane equipped with an automatic cable scraping system.

The diet was prepared as a TMR and offered to calves twice daily at 0800 and $1600 \mathrm{~h}$ for ad libitum intake. Fresh drinking water was freely accessible. Feed bunks were located in front of the lane. The amount of 
feed offered was adjusted daily to ensure at least $10 \%$ orts. Feed offered and refused was recorded daily for each calf. Sample of the feed and orts were taken daily and pooled weekly. All samples were stored at $-20^{\circ} \mathrm{C}$ until analysis.

Experiment 2. Fifteen male Holstein calves (201 \pm $24 \mathrm{~kg} \mathrm{BW}$ ) were randomly assigned to 3 groups with 5 calves per group. The calves in the control group received the basal diet (Table 1) only, whereas calves in the RP-Leu and rumen-protected lysine (RP-Lys) groups were supplemented with $36 \mathrm{~g} / \mathrm{d}$ of RP-Leu and $56 \mathrm{~g} / \mathrm{d}$ of RP-Lys (equal molar to RP-Leu), respectively. The RP-Lys (Hangzhou King Techina Technology Co. Ltd.) contained $45 \%$ lysine within a palm oil coating, which could provide an $80 \%$ rumen-bypass rate in situ and a $98 \%$ abomasal release rate (Rossi et al., 2003). To confirm absorption of the supplemented RP-Leu and RP-Lys, the plasma concentrations of amino acid concentrations were determined, and the concentrations of both leucine and lysine were found to be significantly increased $(P<0.001)$. Housing and feeding regimens of the calves were the same as in experiment 1 .

Experiment 3. Twenty-four male Holstein calves $(289 \pm 26 \mathrm{~kg}$ BW $)$ were randomly allocated into 4 groups with 6 calves per group in a $2 \times 2$ factorial design. The 2 factors were RES (high or low level) and RP-Leu supplementation (0 or $36 \mathrm{~g} / \mathrm{d}$ ). The RES was prepared as coarsely ground corn (high ruminal escape rate) or steam-flaked corn (360 g/L density, low ruminal escape rate). The ingredients and nutritional composition of the basal diet (Table 1) and the housing

Table 1. Ingredients and chemical composition of the experimental diets

\begin{tabular}{lccc}
\hline & & \multicolumn{2}{c}{ Experiment $3^{1}$} \\
\cline { 3 - 4 } Item & $\begin{array}{c}\text { Experiments } \\
\text { 1 and } 2\end{array}$ & L-RES & H-RES \\
\hline Ingredient (\% of DM) & & & \\
Alfalfa hay & 21.5 & 13.9 & 13.9 \\
Oat & 21.5 & 11.5 & 11.5 \\
Corn silage & - & 20.4 & 20.4 \\
Steam-flaked corn & 33.3 & 39.8 & - \\
Coarsely ground corn & - & - & 39.8 \\
Soybean meal & 18.3 & 11.2 & 11.2 \\
Premix & 5.4 & 3.2 & 3.2 \\
Chemical composition & & & \\
DM (\%) & 57.3 & 55.5 & 55.8 \\
CP (\% of DM) & 16.9 & 13.8 & 14.0 \\
Starch (\% of DM) & 23.3 & 33.5 & 33.0 \\
NDF (\% of DM) & 27.4 & 23.5 & 23.5 \\
ADF (\% of DM) & 18.5 & 15.4 & 15.5 \\
\hline${ }^{1}$ L-RES = low rumen-escape starch; H-RES $=$ high rumen-escape \\
starch. \\
${ }^{2}$ Each kilogram contained 1,800 mg of Fe, 350 mg of Cu, 2,160 mg of \\
Mn, 2,660 mg of Zn, 14 mg of Se, 21 mg of I, 18 mg of Co, 180,000 \\
IU of vitamin A, 45,000 IU of vitamin D, and 2,150 IU of vitamin E.
\end{tabular}

and feeding regimens of the calves were the same as in experiment 1 . The diet was formulated to provide adequate ruminally available $\mathrm{N}$ and to meet or exceed the requirements for vitamins and minerals. The diet was specifically designed to contain higher starch content to ensure an adequate amount of starch flowing to the small intestine, and it was also designed to provide only modest amounts of RUP so that MP supply would be moderate.

\section{Sample Collection and Analysis}

Blood samples were taken at 0,2 , and $4 \mathrm{~h}$ postfeeding on the first $2 \mathrm{~d}$ of the sampling period, from the jugular vein into evacuated tubes containing either sodium fluoride and potassium oxalate for glucose analysis or potassium ethylene diamine tetra-acetic acid $\left(\mathrm{K}_{2} \mathrm{EDTA}\right)$ for analysis of plasma insulin and urea nitrogen. Samples were immediately placed on ice and brought back to the laboratory for further processing within $3 \mathrm{~h}$ of the collection. The samples were centrifuged at $1,000 \times g$ for $20 \mathrm{~min}$ at $4^{\circ} \mathrm{C}$. Plasma (the $\mathrm{K}_{2}$ EDTA tubes) or serum (the tubes containing sodium fluoride and potassium oxalate) samples were stored at $-20^{\circ} \mathrm{C}$ until further processing and analysis.

For analyses of whole-tract digestibility, fecal VFA, and microbial composition, fecal samples were collected on the third to fifth days of the sampling period. Acid detergent insoluble ash was used as an internal marker for calculation of digestibility. Fecal sampling times were scheduled over $3 \mathrm{~d}$ so that the samples represented every $3 \mathrm{~h}$ in a 24-h feeding cycle. At each sampling time, an aliquot of each fecal sample was immediately stored in liquid nitrogen, and the remainder was stored at $-20^{\circ} \mathrm{C}$. After sampling, all samples from each calf stored in liquid nitrogen were pooled, mixed, and homogenized using a sterile slap homogenizer. Then, about $1 \mathrm{~g}$ of subsample was taken and frozen at $-80^{\circ} \mathrm{C}$ for metagenomic DNA extraction. The samples stored at $-20^{\circ} \mathrm{C}$ from each calf were also pooled, mixed, and homogenized. A subsample of approximately $4 \mathrm{~g}$ was mixed in $4 \mathrm{~mL}$ of distilled water for VFA extraction and analysis (Mao et al., 2012).

One rumen fluid sample (about $150 \mathrm{~mL}$ ) was collected via esophageal tube from each calf at $2 \mathrm{~h}$ and $4 \mathrm{~h}$ post-feeding on $\mathrm{d} 4$ and $\mathrm{d} 5$ of the sampling period. After discarding the first $50 \mathrm{~mL}$ of fluid to minimize saliva contamination, approximately $100 \mathrm{~mL}$ of rumen fluid was collected. Samples were immediately measured for pH using a portable pH meter (HI 9125; Hanna Instruments, Woonsocket, RI). Then, each sample was filtered through 4 layers of gauze. A subsample of $10 \mathrm{~mL}$ was acidified by adding $2 \mathrm{~mL}$ of $25 \%$ (wt/vol) metaphosphoric acid and stored at $-20^{\circ} \mathrm{C}$ until VFA analysis. 


\section{Chemical and Biochemical Analyses}

Feed and fecal samples were dried for $24 \mathrm{~h}$ at $105^{\circ} \mathrm{C}$ for DM analysis to adjust dietary ingredient inclusion rates and determine DMI. Duplicate composite samples were dried in a hot-air oven at $55^{\circ} \mathrm{C}$ for $48 \mathrm{~h}$ and ground in a Wiley mill with a 2 -mm mesh screen (ThomasWiley Laboratory Mill, Thomas Scientific, Swedesboro, NJ) followed by a 1-mm mesh screen. All the samples were analyzed for $\mathrm{DM}\left(105^{\circ} \mathrm{C}\right.$ for $\left.8 \mathrm{~h}\right)$, CP (method \#988.05), and crude ash (\#942.05) according to AOAC methods (AOAC International, 2002). Diet NDF and ADF were analyzed using sodium sulfite and heat-stable $\alpha$-amylase (Ankom A200I Fiber Analyzer, Ankom Technology, Macedon, NY) according to Van Soest et al. (1991). Starch content was determined through an enzymatic method ( $\alpha$-amylase and amyloglucosidase) with a commercial starch analysis kit (Megazyme International Ireland Ltd., Bray, Ireland). Acid detergent insoluble ash was determined using a modified procedure (method \# 935.29) from the AOAC Methods (AOAC International, 2002). Digestibility was calculated according to acid detergent insoluble ash content (Van Keulen and Young, 1977).

Glucose (Barham and Trinder, 1972) and plasma urea nitrogen (Rahmatullah and Boyde, 1980) were analyzed using commercial kits (Jiancheng Bioengineering Institute, Nanjing, China) based on enzymatic colorimetric methods, and serum insulin was determined using an ELISA kit (bovine insulin ELISA kit, catalog no. E11I0004; Cloud-Clone Corporation, Houston, TX). The VFA concentrations in the rumen fluid and the fecal samples were analyzed using a gas chromatograph (Agilent Technologies 7820A GC system, Santa Clara, $\mathrm{CA})$ equipped with a $30-\mathrm{m} \times 0.25-\mathrm{mm} \times 0.33-\mu \mathrm{m}$ fused silica column (AE-FFAP; ATECH Technologies Co. Ltd., Shanghai, China) after removing the solid particles and protein in the sample according to Li et al. (2014).

\section{Illumina MiSeq Sequencing and Sequence Analysis of Fecal Microbiota}

Metagenomic DNA was extracted from fecal samples using the EZNA soil DNA Kit (Omega Bio-Tek, Norcross, GA) according to the manufacturer's protocols. The final DNA concentration and purity were determined using a NanoDrop 2000 UV-vis spectrophotometer (Thermo Fisher Scientific, Waltham, MA), and DNA quality was visually assessed using agarose gel (1\%) electrophoresis. The V3-V4 hypervariable regions of the bacterial $16 \mathrm{~S}$ rRNA gene were amplified with primers 338F (5'-ACTCCTACGGGAGGCAGCAG- $3^{\prime}$ ) and 806R (5'-GGACTACHVGGGTWTCTAAT-3') us- ing a thermocycler PCR system (GeneAmp 9700, Applied Biosystems, Thermo Fisher Scientific). The PCR reactions were conducted using the following program: $3 \mathrm{~min}$ of denaturation at $95^{\circ} \mathrm{C}, 27$ cycles of $30 \mathrm{~s}$ at $95^{\circ} \mathrm{C}, 30 \mathrm{~s}$ for annealing at $55^{\circ} \mathrm{C}, 45 \mathrm{~s}$ for elongation at $72^{\circ} \mathrm{C}$, and a final extension at $72^{\circ} \mathrm{C}$ for $10 \mathrm{~min}$. The PCR reactions were performed in triplicate in $20 \mu \mathrm{L}$ mixture containing $4 \mu \mathrm{L}$ of $5 \times$ FastPfu (TransGen Biotech, Beijing, China) buffer, $2 \mu \mathrm{L}$ of $2.5 \mathrm{~m} M$ dNTP, $0.8 \mu \mathrm{L}$ of each primer $(5 \mu M), 0.4 \mu \mathrm{L}$ of FastPfu polymerase, and $10 \mathrm{ng}$ of template DNA. The resulting PCR products were first gel purified using a $2 \%$ agarose gel and the AxyPrep DNA Gel Extraction Kit (Axygen Biosciences, Union City, CA) and quantified using QuantiFluor-ST (Promega, Madison, WI) according to the manufacturer's protocol. Purified amplicons were pooled in equimolar and paired-end sequenced $(2 \times 300)$ on an Illumina MiSeq platform (Illumina, San Diego, CA) according to standard protocols by Majorbio Bio-Pharm Technology Co. Ltd. (Shanghai, China). The raw sequencing reads were deposited into the NCBI Sequence Read Archive database (Accession Number: PRJNA548622).

Raw FASTQ files were demultiplexed, quality-filtered using Trimmomatic (http://www.usadellab.org/ cms/index.php?page $=$ trimmomatic), and merged using FLASH (https://ccb.jhu.edu/software/FLASH/) with the following criteria. (1) The reads were truncated at any site receiving an average quality score $<20$ over a 50-bp sliding window. (2) The merged reads had identical barcode and no more than 2 nucleotide mismatches in the primer regions, and reads containing ambiguous bases were removed. (3) The reads whose overlap was longer than $10 \mathrm{bp}$ were merged according to their overlap sequence. Operational taxonomic units were clustered with a 97\% similarity cutoff using UPARSE (version 7.1, http://drive5.com/uparse/), and chimeric sequences were identified and removed using UCHIME (http://www.drive5.com/uchime/). The taxonomy of each operational taxonomic unit was assigned by classifying its representative sequence using the RDP Classifier algorithm (http://rdp.cme.msu.edu/) against the Silva 16S rRNA database (SSU123; https://www.arb -silva.de/) using a confidence threshold of $70 \%$.

\section{Statistical Analysis}

All statistical analyses were performed using SAS version 9.1 (SAS Institute Inc., Cary, NC). In experiments 1 and 2, the PROC GLM procedure for a single measure or repeated measures was used to determine the differences in all the measures between the control and the treatments. One calf in experiment 2 died due 
to rumen distension, and its data were excluded from the statistical analysis.

Experiment 3 included 2 factors: RP-Leu and RES, and thus the data were analyzed using the MIXED procedure, including the interaction between the 2 factors, with sampling time being included as a repeated factor. When the interaction was significant, Tukey's multiple comparison test was used to assess the differences between treatment means. Results were expressed as least squares means and standard errors of the mean. Treatment differences were declared significant when $P$ $<0.05$, and tendencies were declared when $0.05 \leq P$ $\leq 0.10$.

\section{RESULTS AND DISCUSSION}

To assess starch digestibility and utilization in the small intestine, we proposed a model for indirect estimation, as shown in Figure 1. Starch digestion in ruminants occurs in the 3 major compartments of the digestive tract: the rumen, small intestine, and hindgut. Starch degradation in the rumen can be evaluated via the rumen VFA profile, because starch is fermented rapidly in the rumen, with increasing propionate production. Much of the rumen-bypass starch is digested and absorbed as glucose in the small intestine, and the remaining starch can be fermented in the hindgut, also with propionate or butyrate as major fermentation products. Propionate is the major substrate for gluconeogenesis in ruminants, and thus a change of blood glucose concentration can serve as a proxy of starch digestibility in the rumen compared with the small intestine. Lactic acid is another substrate for gluconeogenesis in ruminants, but it was not included in the model, because its concentration is many orders of magnitude lower than that of propionate. Starch digestion in the hindgut can be evaluated via the fecal VFA profile in combination with the microbial profile (Figure 1). It should be noted that the model is for indirect estimation of starch digestion and utilization, and it relies on the relative changes in these indicators between the control and the treatment. This model does not allow an estimate of the absolute values of starch digestion.

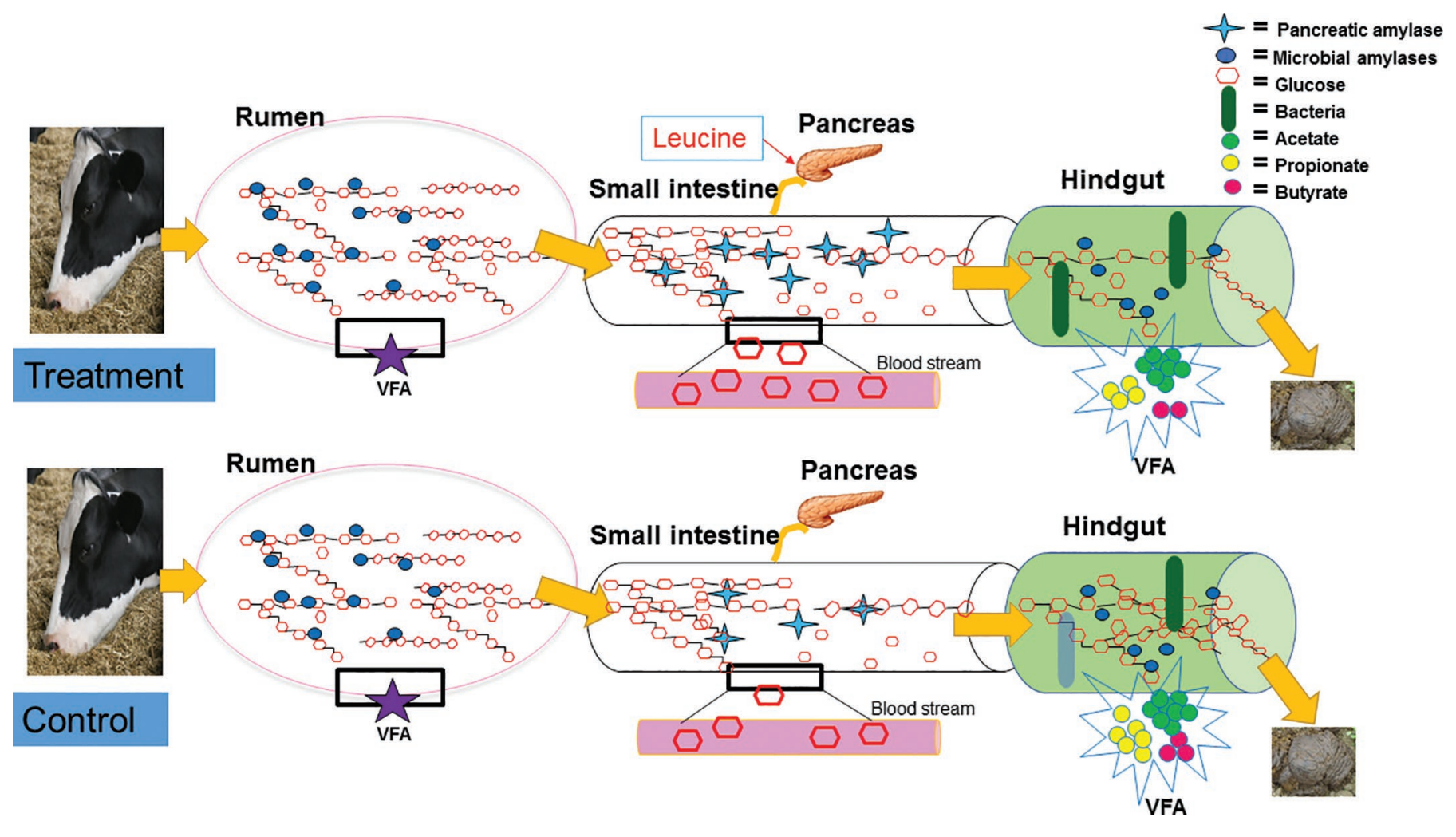

Figure 1. A static model to illustrate starch digestion in the rumen, small intestines, and hindgut. The model is based on the premise that starch digestion in the rumen can be evaluated by examining ruminal VFA profiles, including the molar proportion of propionate (major gluconeogenesis substrate in ruminants), whereas digestion of rumen bypass starch in the small and hindgut intestines can be evaluated by changes in blood glucose concentration and alteration of propionate molar proportion in both the rumen and the feces. Lactic acid (another substrate for gluconeogenesis) in the rumen is not included, because its concentration is many orders lower than that of propionate. Starch digestion in the hindgut can be evaluated by fecal VFA profiles in combination with fecal microbial profiles. 
Experiment 1: RP-Leu Might Improve Small Intestinal Starch Digestion in dairy Calves

Increased Blood Glucose Concentration. In this experiment, apparent whole-tract digestibility of starch averaged 96.36 and $97.35 \%(P=0.411)$ for the control and RP-Leu groups, respectively (Table 2). However, RP-Leu supplementation significantly $(P=$ 0.027) increased blood glucose concentration (Table 3). Both absorption of exogenous glucose and endogenous gluconeogenesis contribute to blood glucose concentration, and the endogenous synthesis is largely from hepatic gluconeogenesis (Nocek and Tamminga, 1991). Ruminants have an obligate need for glucose, and propionate is the primary precursor for gluconeogenesis in fed animals, whereas other fermentation products, such as glycerol, lactate, and amino acids, become important in underfed or fasted animals (Bergman et al., 1968; Lomax and Baird, 1983). The contribution of amino acids to gluconeogenesis in ruminants is minimal (Ørskov and MacLeod, 1990) and might be easily substituted by the provision of other precursors, especially propionate, which is constantly produced during rumen fermentation (Lobley, 1992). The ruminal VFA profile was not affected by RP-Leu (Table 4); hence, the increased blood glucose might be mainly attributable to absorption of exogenous glucose from the small intestine, indicating that RP-Leu might have increased starch digestibility in the small intestine. The similar rumen fermentation profile between the control and the RP-Leu groups also suggests that RP-Leu probably did not affect the rumen fermentation pattern, neither the amounts of starch degraded in the rumen nor the amount flowing into the small intestine. Therefore, the starch fermentation pattern in the hindgut could indirectly reflect starch digestion in the small intestine, and thus we further analyzed the VFA profile and microbial composition in the feces.

Increased Fecal pH, Decreased Fecal Propionate Proportion, and Changed Cellulolytic and Amylolytic Microbial Composition. Fecal VFA concentration reflects the amount of VFA produced by colonic fermentation that has not been absorbed by colonic epithelial cells. Fecal pH is largely influenced by the amount of fermentable substrate degraded in the cecum and colons (Ulyatt et al., 1975). Fecal excretion of VFA could be taken as an indicator for starch fermentation in the hindgut. The RP-Leu group had higher fecal $\mathrm{pH}$ than the control group $(P=0.008)$, but their fecal VFA concentrations were not different (Table 5). Given the negative correlation between fecal $\mathrm{pH}$ and fecal starch content (Nocek, 1997), the higher fecal pH in the RPLeu group might reflect the decreased amount of starch fermented in the hindgut and increased starch digestion in the small intestine in response to RP-Leu supplementation. Furthermore, starch fermentation typically increases propionate and butyrate proportions (Mao et al., 2012), and the decreased propionate proportion in the RP-Leu group $(P=0.013)$ also might indicate reduced starch fermentation in the hindgut of calves supplemented with RP-Leu. Although propionate and butyrate are primary indicators for starch fermentation in the hindgut, acetate is still the dominant end product. Therefore, we should carefully evaluate the fecal propionate and butyrate changes, and fecal amylolytic and cellulolytic microbiota changes might be a more accurate accompanied indicator in response to starch fermentation in the hindgut.

Fermentable substrates in the hindgut could influence fecal microbial composition, and changes in relative abundance of microbial populations can, in turn, reflect the major fermentable substrates available. As shown in Figure 2, RP-Leu increased $(P<0.05)$ the abundance of several cellulolytic genera, such as Pseudobutyrivibrio, Ruminiclostridium, and Lachnospiraceae NK3A20, a finding consistent with a previous report (Yoav et al., 2017). Faecalibacterium is a genus of butyrate-producing bacteria that can utilize starch (Oikonomou et al., 2013), and the decreased level of Faecalibacterium $(P=0.038)$ concurs with the decreased fecal butyrate proportion. Therefore, the increased cellulolytic genera but decreased amylolytic genera in the RP-Leu group mirror the changes in fiber and starch degradation, respectively, and again suggest decreased starch fermentation in the hindgut in the RP-Leu group. The similar whole-tract digestibility of starch and rumen fermentation profile and increased blood glucose concentration in the RP-Leu group compared with the control group further indicate that RP-Leu supplementation might improve starch digestion in the small intestine. Then, a new question could be asked: Could the effects of leucine on intestinal starch digestibility be attributed to its role as a signaling amino acid or as a substrate for protein synthesis? Experiment 2 was conducted to address this question.

\section{Experiment 2: RP-Leu Might Improve Starch Digestion in the Small Intestine, Possibly Beyond Serving as a Precursor of Protein}

No significant difference was observed in the wholetract digestibility of starch (Table 2), plasma insulin and blood urea nitrogen concentrations, or rumen VFA profile among the control, RP-Leu, and RP-Lys groups in experiment 2 (Table 4). Blood glucose concentration was significantly higher only in the RP-Leu group $(P=$ 0.042) compared with those of the RP-Lys and control groups (Table 3 ). 
Ren et al.: LEUCINE INCREASES STARCH DIGESTION IN CALF SMALL INTESTINE
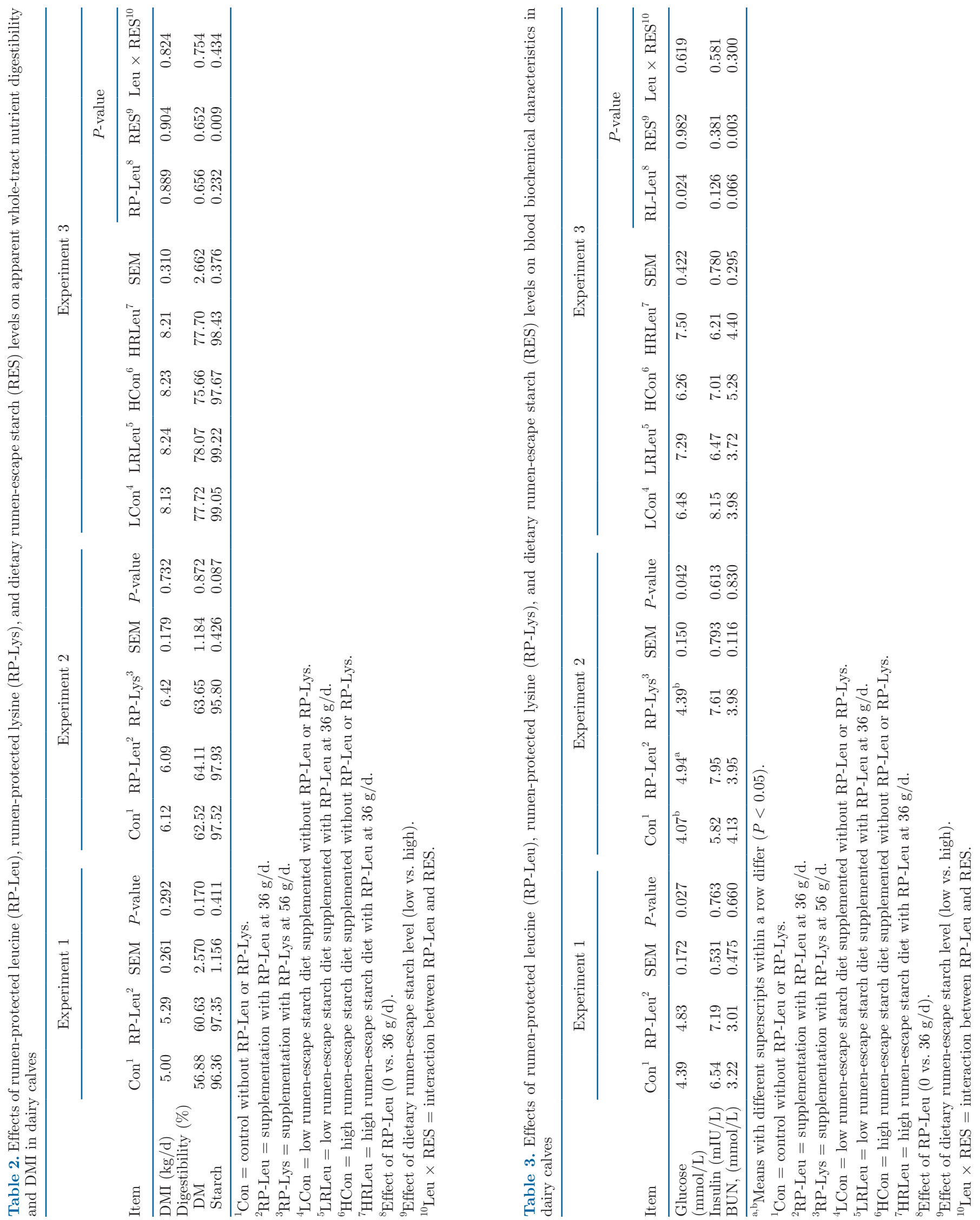
Ren et al.: LEUCINE INCREASES STARCH DIGESTION IN CALF SMALL INTESTINE

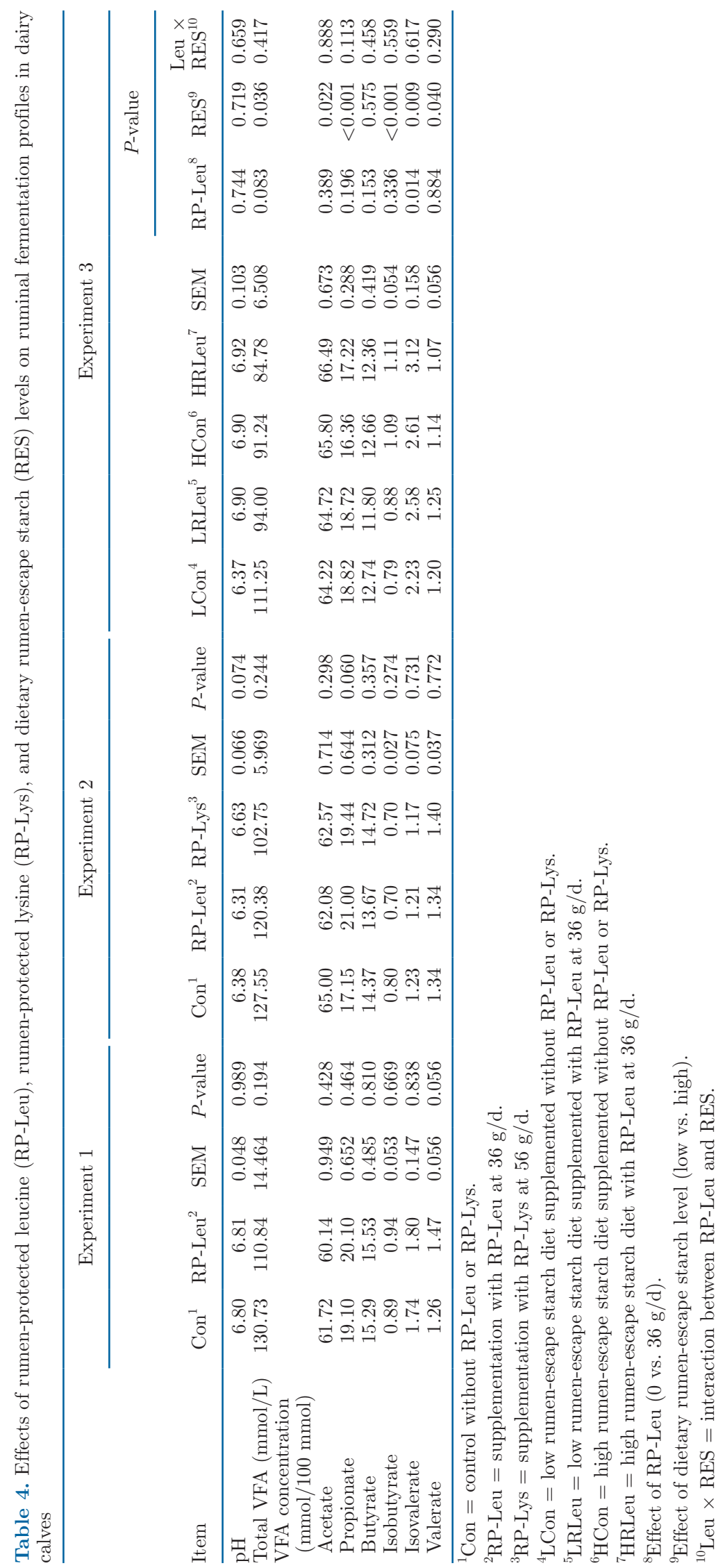


Ren et al.: LEUCINE INCREASES STARCH DIGESTION IN CALF SMALL INTESTINE

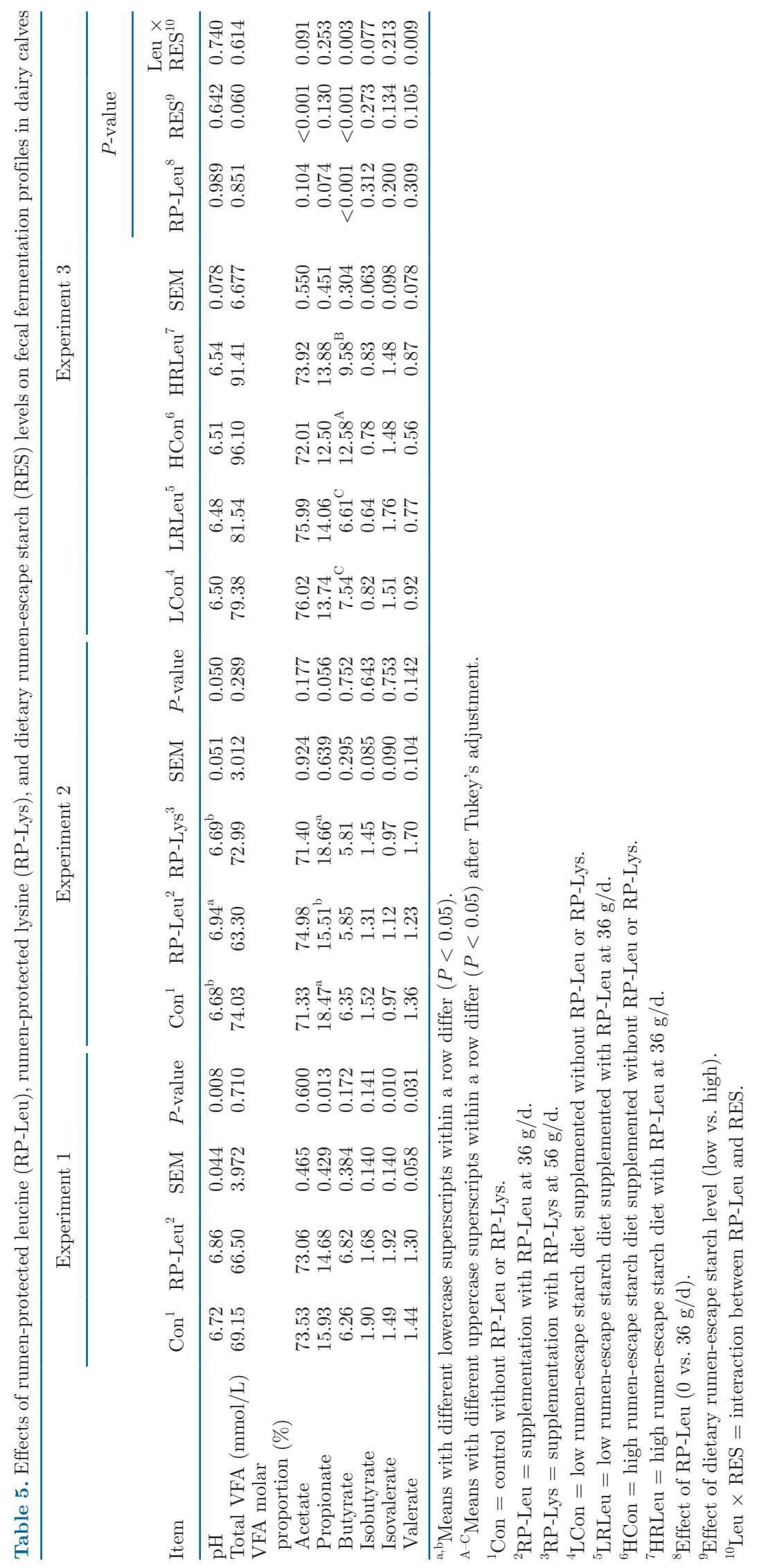



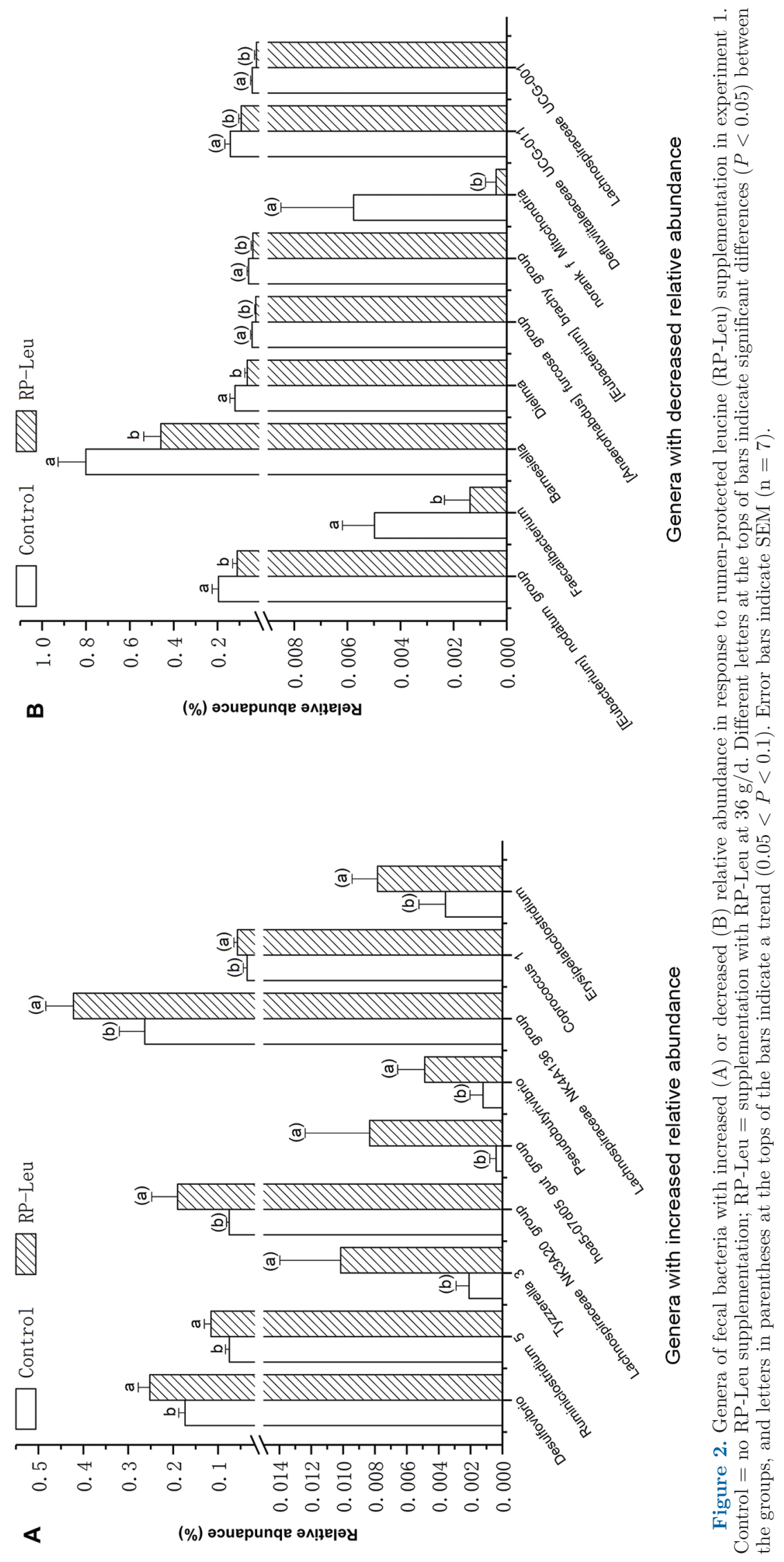
The fecal $\mathrm{pH}$ and propionate proportions were significantly higher in the RP-Leu group than in the RP-Lys and control groups $(P=0.050$; Table 5). Significant differences in fecal microbial population structure at the family (Figure 3) and genus (Figure 4) levels were detected among the 3 groups. The family Ruminococcaceae $(P=0.061)$, which contains several known species of fibrolytic bacteria (Russell and Rychlik, 2001; Liu et al., 2016), and Bacteroidales BS11 gut group $(P=0.062)$, which is capable of digesting complex plant polysaccharides (Meale et al., 2016), tended to be higher in relative abundance in the RP-Leu group than in the RP-Lys or control groups. These results collectively suggest that cellulolysis was increased in the large intestine of the RP-Leu group, whereas the amount of starch digested was decreased, and RP-Lys supplementation had no such effects.

In ruminants, lysine and methionine are 2 limiting amino acids for production (Rogers et al., 1989; Kung and Rode, 1996), and lysine is often regarded as one of the most limiting amino acids for growing cattle (Richardson and Hatfield, 1978), particularly in diets with high proportions of maize products (Williams et al., 1999; Xue et al., 2011). Methionine has several metabolic roles other than serving as a precursor for protein synthesis (methyl donor for transmethylation reactions; Kung and Rode, 1996). Thus, we used lysine, a pure precursor for protein synthesis, as the control, which might exclude the potential roles of leucine in promoting the pancreatic amylase synthesis and secretion as substrate effect. The results of experiments 1 and 2 were consistent with respect to the roles of RP-Leu in modulating starch digestion in the small intestine. However, RP-Lys had no such effects. Some amino acids can serve not only as substrates for protein synthesis but also as signal molecules (Wu, 2013). Leucine can enhance protein synthesis by activating the mTOR pathway as a signal molecule (Anthony et al., 2000;

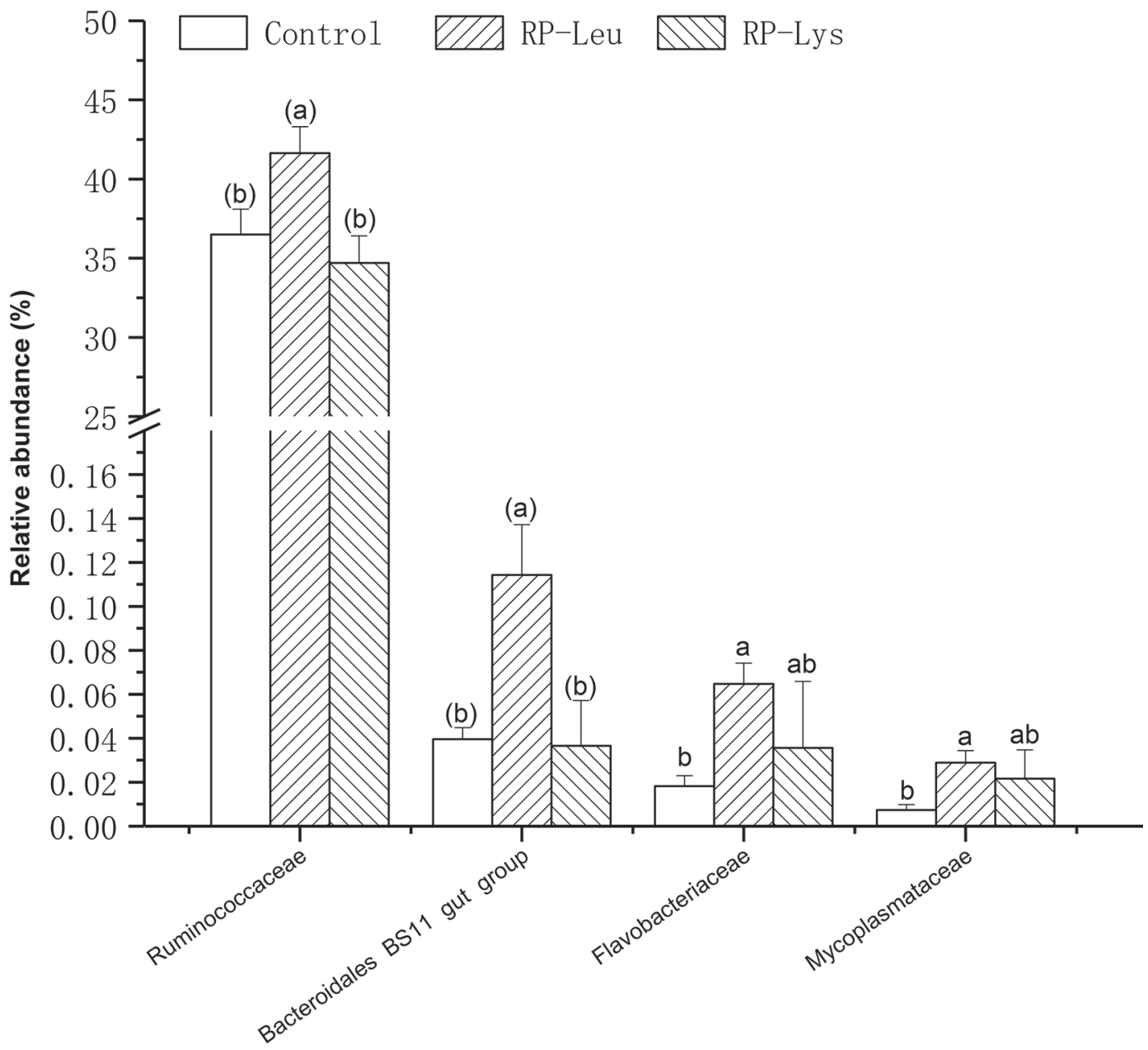

Figure 3. Effects of rumen-protected leucine (RP-Leu) and rumen-protected lysine (RP-Lys) on families of fecal microbiota in experiment 2 . Control = no RP-Leu or RP-Lys supplementation; RP-Leu = supplementation with RP-Leu at $36 \mathrm{~g} / \mathrm{d}$; RP-Lys = supplementation with RP-Lys at $56 \mathrm{~g} / \mathrm{d}$. Different letters at the tops of bars indicate significant differences $(P<0.05)$ between the groups, and letters in parentheses at the tops of bars indicate a trend $(0.05<P<0.1)$. Error bars indicate SEM $(\mathrm{n}=5)$. 


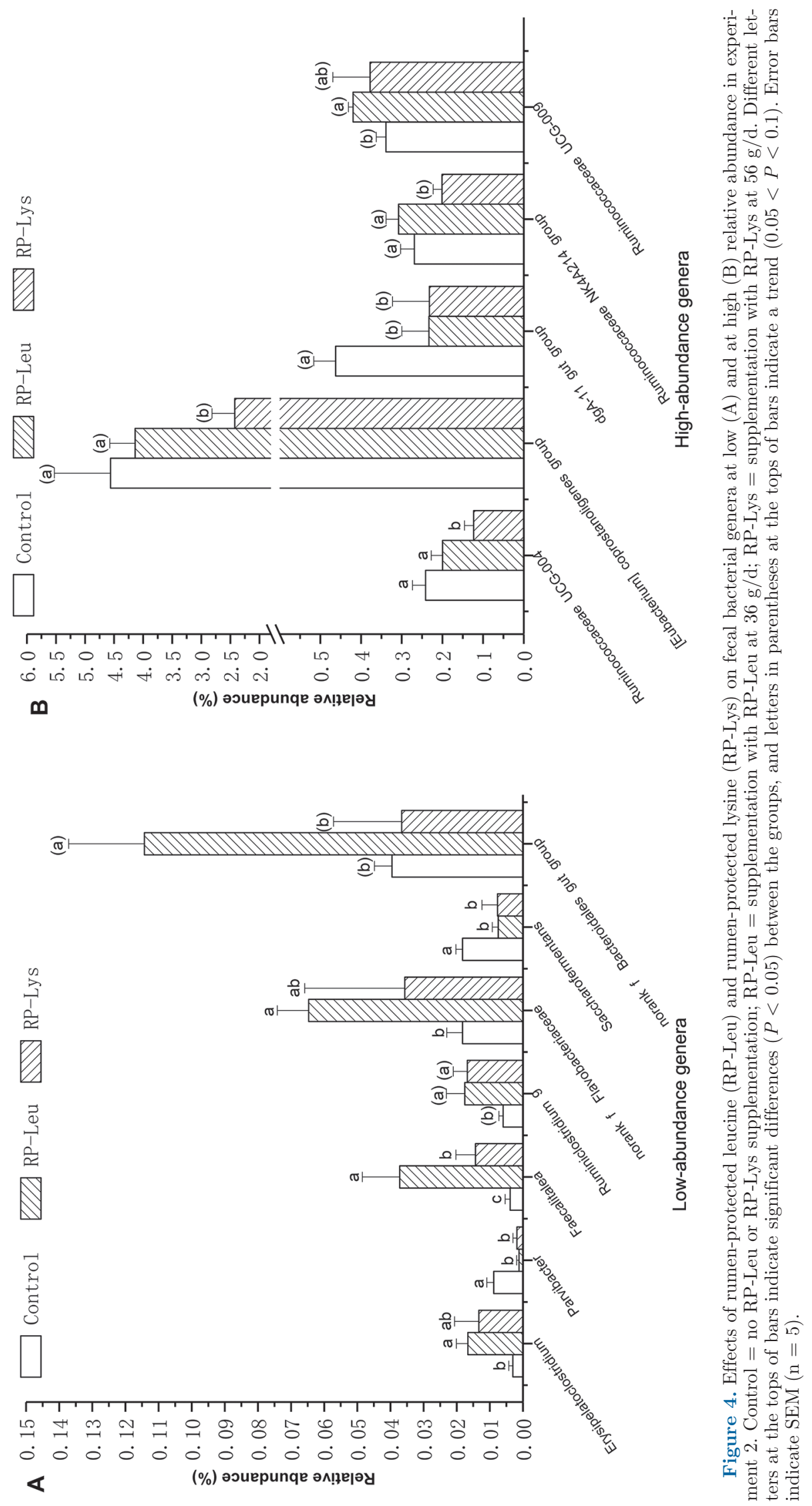


Guo et al., 2018). Indeed, in lactating cows infused with a complete EAA mix, EAA mix without leucine, or EAA without lysine, the mammary abundance of phosphorylated S6K1, an indicator of the mTORC 1 activity, was found not to be affected by the EAA mix, but it was increased by the EAA mix without lysine, and milk protein was significantly lower when leucine, but not lysine, was excluded from the EAA mix (Doelman et al., 2015). These results suggest that leucine, but not lysine, can enhance milk protein synthesis via the mTOR pathway. Another study also showed the inability of lysine to stimulate protein synthesis as a singling molecule (Sato et al., 2014). On the basis of the results in the literature and the present study, we proposed that RP-Leu, but not RP-Lys, might improve starch digestion in the small intestine by regulating, possibly beyond serving as a precursor of protein, the signaling pathways involved in pancreatic amylase protein synthesis. Because we were unable to directly prove that RP-Leu was acting as signal molecule in the present study, future research involving pancreas biopsy and analysis of the phosphorylation of the mTOR pathway is needed to elucidate the mechanism by which leucine regulates pancreatic acinar amylase synthesis as a signal molecule.

\section{Experiment 3: Interactions Between RP-Leu and Post-Ruminal Starch Levels Implied the Small Intestine as the Site of RP-Leu Effect}

RES Affected Apparent Total-Tract Starch Digestibility and Rumen Fermentation Profiles. The levels of RES in the diets were modulated by different processing of corn: steam-flaking for low-RES or coarsely grinding for high-RES. The low-RES diet resulted in greater $(P=0.009)$ whole-tract digestibility of starch than did the high-RES diet (Table 2). Improvements in whole-tract starch digestibility by steam flaking have been reported previously (Corona et al., 2005; Zhong et al., 2008; Zhou et al., 2015; Luo et al., 2017). Steam flaking, which is a combination of heat, moisture, and mechanical actions, disrupts the endosperm structure, exposes the starch granules, causes varying degrees of starch gelatinization, and allows ruminal microorganisms increased access to amylose and amylopectin molecules (Kotarski et al., 1992), thereby improving starch digestion in the rumen (Huntington, 1997). Consistent with the results from previous studies (Zinn, 1987; Lesmeister and Heinrichs, 2004), we found that the low-RES diet increased total ruminal VFA concentration $(P=0.036)$ and propionate molar proportion $(P<0.001)$, while decreasing $(P<0.022)$ the molar proportions of acetate and isobutyrate (Table $4)$.

Journal of Dairy Science Vol. 103 No. 5, 2020
Using the relationship between DMI and rumen starch degradation rate developed by Luo et al. (2017), we estimated the amounts of starch flowing into the small intestine for the high-RES $(1.023 \mathrm{~kg} / \mathrm{d})$ and the low-RES groups $(0.765 \mathrm{~kg} / \mathrm{d})$. The amount of starch was significantly higher in the high-RES group than in the low-RES group. Rumen starch degradation rates for the corn types were determined in situ using the nylon bag technique from Luo et al. (2017), and the equation calculating rumen starch degradation rate assumed an average passage rate of $0.06 / \mathrm{h}$ (Ørskov and McDonald, 1979; Offner et al., 2003). The different DMI between cows and calves might have little influence on the rumen starch degradation rate of steam-flaked corn or fine-ground corn.

According to a kinetic-based model describing small intestinal digestion of starch, the small intestine digestion was only above $70 \%$ when $700 \mathrm{~g} / \mathrm{d}$ of starch or less reaches the small intestine (Huntington et al., 2006). In another study, apparent small intestinal starch disappearance was $56 \%$ when steers were abomasally infused $1,042 \mathrm{~g} / \mathrm{d}$ cornstarch (Richards et al., 2002). However, Westreicher-Kristen et al. (2018) reported that starch disappearance from the small intestine averaged $85 \%$ when $880 \mathrm{~g} / \mathrm{d}$ starch was abomasally infused in heifers, and further decreased from 78 to $68 \%$ at intestinal supplies of 953 and 1,993 g/d of starch, respectively (Robbers et al., 2019). When considering the results from different experiments, we must consider the differences between experiments, such as the animal, BW, feed intake, diet, and starch sources, as well as the experimental design (e.g., time for adaption to starch digestion). In our study, more than $700 \mathrm{~g} / \mathrm{d}$ of starch reached the small intestine in the growing dairy calves, which suggests that adequate starch flowed to the small intestine.

In addition, RES levels might have changed the microbial protein and bypass protein flowing into the small intestine, which could potentially influence the effects of leucine noted in the present study. We used the CPM Dairy software (https://cpm-dairy.updatestar .com/en) to predict the metabolizable protein and duodenal amino acid flows for the high- and low-RES groups; the differences between these 2 groups in the total or bacterial amino acid flows were less than $1 \mathrm{~g} / \mathrm{d}$, less than $1 \%$ of the total flow. Such a small difference might be too small to make a significant contribution to the difference in starch digestion between the 2 groups. We therefore proposed that the difference in the starch digestion in the small intestine was primarily due to the role of RP-Leu.

Effect of RP-Leu Was Positively Affected by Postruminal Dietary Starch Levels. No significant effects of RP-Leu were detected on the whole-tract 
Table 6. Effects of rumen-protected leucine (RP-Leu) supplementation (0 or $36 \mathrm{~g} / \mathrm{d}$ ) and dietary rumen-escape starch (RES) levels on the relative abundance $(\%)$ of fecal bacterial phylum of the calves in experiment 3

\begin{tabular}{|c|c|c|c|c|c|c|c|c|}
\hline \multirow[b]{2}{*}{ Item } & \multicolumn{2}{|c|}{ Low RES } & \multicolumn{2}{|c|}{ High RES } & \multirow[b]{2}{*}{ SEM } & \multicolumn{3}{|c|}{$P$-value } \\
\hline & 0 & 36 & 0 & 36 & & RP-Leu & RES & Leu $\times$ RES \\
\hline Proteobacteria & 2.82 & 1.55 & 10.92 & 8.76 & 1.693 & 0.324 & $<0.001$ & 0.796 \\
\hline Spirochaetae & 9.52 & 3.53 & 4.13 & 2.08 & 1.950 & 0.052 & 0.095 & 0.325 \\
\hline Tenericutes & 1.37 & 1.80 & 1.59 & 1.09 & 0.256 & 0.902 & 0.356 & 0.087 \\
\hline Others & 0.29 & 0.21 & 0.22 & 0.14 & 0.069 & 0.244 & 0.338 & 0.981 \\
\hline
\end{tabular}

$\overline{\mathrm{A}, \mathrm{B}}$ Different superscripts in the same row indicate significant difference $(P<0.05)$ after Tukey's adjustment.

digestibility of starch (Table 2), blood insulin (Table $3)$, rumen total VFA concentration, or VFA profiles, except for increased isovalerate molar proportion $(P$ $=0.014$; Table 4). However, RP-Leu supplementation increased blood glucose concentration $(P=0.024)$ and tended to reduce BUN concentration $(P=0.066)$. The high-RES diet reduced starch digestibility $(P=0.066$; Table 2) and increased blood BUN concentration $(P$ $=0.003 ;$ Table 3$)$, total rumen VFA concentration $(P=0.036)$, and molar proportion of propionate and valerate $(P<0.01)$ while increasing the molar proportion of isobutyrate and isovalerate $(P=0.005$; Table 4) compared with the low-RES diet. Without RP-Leu supplementation, the high- and low-RES groups had similar blood glucose and insulin concentrations $(P>$ 0.05; Table 3). The RP-Leu supplementation resulted in significant increases in blood glucose concentration in both the high- and low-RES groups, but at 2 different magnitudes: $19.8 \%$ versus $12.5 \%$ increase. Apparently, the stimulating effect of RP-Leu on starch digestion was positively correlated with the amount of starch reaching the small intestine.

Interactions Between RP-Leu and Postruminal Starch Levels Affected the Fecal VFA Profile and Microbiota. As shown in Table 5, the high-RES diet resulted in a high butyrate concentration in feces,

Table 7. Effects of rumen-protected leucine (RP-Leu) supplementation (0 or $36 \mathrm{~g} / \mathrm{d}$ ) and dietary rumen-escape starch (RES) levels on the relative abundance $(\%)$ of fecal bacterial families in the calves in experiment 3

\begin{tabular}{|c|c|c|c|c|c|c|c|c|}
\hline \multirow[b]{2}{*}{ Item } & \multicolumn{2}{|c|}{ Low RES } & \multicolumn{2}{|c|}{ High RES } & \multirow[b]{2}{*}{ SEM } & \multicolumn{3}{|c|}{$P$-value } \\
\hline & 0 & 36 & 0 & 36 & & Leu & RES & Leu $\times$ RES \\
\hline Ruminococcaceae & 32.34 & 35.55 & 26.86 & 25.33 & 2.204 & 0.709 & 0.002 & 0.294 \\
\hline Lachnospiraceae & 16.13 & 15.53 & 19.94 & 23.48 & 2.476 & 0.558 & 0.028 & 0.413 \\
\hline Prevotellaceae & 10.34 & 7.55 & 6.27 & 7.52 & 2.236 & 0.733 & 0.371 & 0.377 \\
\hline Bacteroidaceae & 5.99 & 7.86 & 10.81 & 5.71 & 1.60 & 0.325 & 0.412 & 0.041 \\
\hline Succinivibrionaceae & 2.51 & 1.42 & 10.53 & 8.52 & 1.680 & 0.367 & $<0.001$ & 0.785 \\
\hline Rikenellaceae & 7.82 & 9.61 & 4.19 & 3.76 & 1.712 & 0.695 & 0.012 & 0.526 \\
\hline Spirochaetaceae & 9.52 & 3.53 & 4.13 & 2.08 & 1.950 & 0.052 & 0.095 & 0.325 \\
\hline Bacteroidales $\mathrm{S} 24-7$ group & 4.31 & 4.48 & 5.70 & 5.21 & 0.798 & 0.847 & 0.201 & 0.684 \\
\hline Clostridiaceae_1 & 0.32 & 0.31 & 2.40 & 1.43 & 0.395 & 0.227 & 0.001 & 0.241 \\
\hline Erysipelotrichaceae & 0.83 & 1.27 & 1.39 & 1.05 & 0.206 & 0.814 & 0.410 & 0.074 \\
\hline Acidaminococcaceae & 0.93 & 0.76 & 1.14 & 1.00 & 0.245 & 0.540 & 0.360 & 0.943 \\
\hline Christensenellaceae & 0.94 & 1.03 & 0.82 & 1.12 & 0.162 & 0.254 & 0.917 & 0.514 \\
\hline Norank_o_Mollicutes RF9 & 0.88 & 0.70 & 0.59 & 0.71 & 0.123 & 0.810 & 0.275 & 0.225 \\
\hline Clostridiales vadinBB60 group & 0.72 & 0.88 & 0.52 & 0.71 & 0.158 & 0.279 & 0.252 & 0.918 \\
\hline Peptostreptococcaceae & 0.83 & 0.40 & 0.53 & 0.70 & 0.116 & 0.282 & 0.986 & 0.017 \\
\hline Bifidobacteriaceae & 0.66 & 0.17 & 0.51 & 0.25 & 0.305 & 0.230 & 0.907 & 0.713 \\
\hline Bacteroidales incertae sedis & 0.53 & 0.53 & 0.44 & 0.18 & 0.167 & 0.456 & 0.205 & 0.426 \\
\hline unclassified_o_Bacteroidales & 0.23 & 0.30 & 0.35 & 0.45 & 0.126 & 0.514 & 0.298 & 0.886 \\
\hline Porphyromonadaceae & 0.97 & 0.38 & 0.21 & 0.14 & 0.318 & 0.311 & 0.132 & 0.423 \\
\hline Veillonellaceae & 0.32 & 0.23 & 0.36 & 0.60 & 0.128 & 0.587 & 0.117 & 0.219 \\
\hline Bacteroidales RF16 group & 0.89 & 0.50 & 0.13 & 0.01 & 0.243 & 0.304 & 0.017 & 0.580 \\
\hline Anaeroplasmataceae & 0.25 & 0.38 & 0.49 & 0.18 & 0.138 & 0.510 & 0.894 & 0.122 \\
\hline Norank_o_EMP-G18 & 0.14 & 0.62 & 0.45 & 0.17 & 0.140 & 0.491 & 0.619 & 0.013 \\
\hline Norank_o_Gastranaerophilales & $0.19^{\mathrm{A}}$ & $0.01^{\mathrm{B}}$ & $0.04^{\mathrm{B}}$ & $0.04^{\mathrm{B}}$ & 0.034 & 0.019 & 0.080 & 0.015 \\
\hline Others & 1.42 & 1.07 & 1.21 & 1.16 & 0.145 & 0.193 & 0.691 & 0.316 \\
\hline
\end{tabular}

$\overline{\mathrm{A}, \mathrm{B}}$ Different superscripts in the same row indicate significant difference $(P<0.05)$ after Tukey's adjustment. 
whereas RP-Leu supplementation decreased the fecal butyrate molar proportion $(P<0.001)$. Significant interactions were noted between RP-Leu and RES levels with respect to the molar proportion of fecal butyrate and valerate $(P<0.05)$, which suggests that the influence of RES levels on butyrate and valerate was affected by RP-Leu supplementation. Examining the effects of RES levels, Ørskov et al. (1969) found that cecal butyrate molar proportion averaged 5.8 and $19.4 \%$ when sheep were fed barley- and maize-based diets, respectively. Similarly, a 3-fold increment of butyrate in the colon, cecum, and feces was observed when starch was infused into the terminal ileum of sheep (Ørskov et al., 1970). Additionally, fecal butyrate excretion increased linearly with increasing amounts of corn starch infused into the abomasum of heifers

Table 8. Effects of rumen-protected leucine (RP-Leu) supplementation (0 or $36 \mathrm{~g} / \mathrm{d}$ ) and dietary rumen-escape starch (RES) levels on the relative abundance $(\%)$ of fecal bacterial genera in the calves in experiment 3

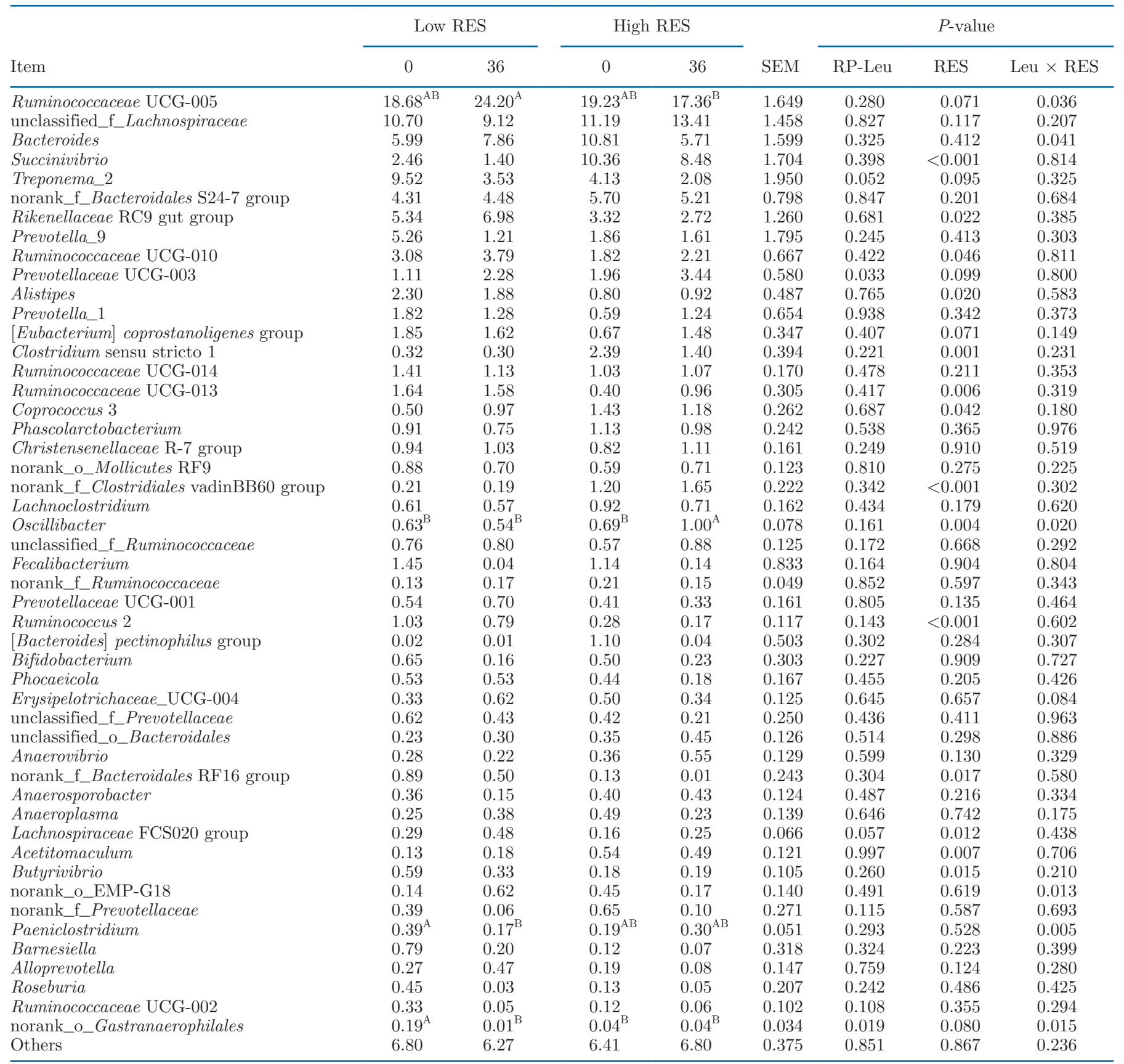

$\overline{\mathrm{A}, \mathrm{B}}$ Different superscripts in the same row indicate significant difference $(P<0.05)$ after Tukey's adjustment. 
(Robbers et al., 2019). Therefore, a decreased molar proportion of butyrate in feces might indicate a decreased amount of starch available for fermentation in the hindgut. In the present study, the fecal butyrate molar proportion decreased by 23.85 and $12.33 \%$ in the high- and the low-RES groups, respectively, with RPLeu supplementation compared with the control group, suggesting that the RP-Leu effect was more profound at the high-RES level, which was consistent with blood glucose concentrations described earlier. The reduction of fecal butyrate excretion indicates that starch digestion in the small intestine was increased by RP-Leu supplementation. As for fecal propionate excretion, contrary to the results of experiments 1 and 2, which showed a significant decrease in fecal excretion in the RP-Leu group, experiment 3 showed a tendency for fecal propionate excretion to increase $(P=0.074)$ in the RP-Leu group compared with the control group. The higher dietary starch content in experiment 3 than in experiments 1 and 2 might have increased the amount of glucose (and its disaccharides and trisaccharides) flowing to the hindgut due to the limitation of glucose absorption in the small intestine (Westreicher-Kristen et al., 2018), resulting in an increased hindgut fermentation and molar proportion of propionate.

The high-RES diet decreased the relative abundance of the phylum Bacteroidetes $(P=0.046)$ but increased the abundance of Proteobacteria $(P<0.001)$ in the fecal microbiota (Table 6). An interaction was noted between RP-Leu and RES level with respect to the relative abundance of the family Bacteroidaceae $(P=$ 0.041). We found that RP-Leu decreased the relative abundance of Bacteroidaceae in the fecal microbiota of the calves fed the high-RES but not the low-RES diets, suggesting that the effect of RP-Leu was affected by the amounts of starch flowing into the small intestine. Supplementation with RP-Leu tended to increase the relative abundance of Firmicutes $(P=$ 0.072 ), which is positively correlated with dietary fiber content (Trompette et al., 2014). The decreased relative abundance of the family Ruminococcaceae $(P$ $=0.002$ ) corresponding to the high-RES diet (Table 7 ) was consistent with the results of experiment 2, indicating that RP-Leu could improve small intestinal starch digestion when RES was increased. The high-RES diet increased the relative abundance of Lachnospiraceae $(P=0.028)$ and Succinivibrionaceae $(P<0.001)$, both of which have amylolytic species (Mao et al., 2013; Henderson et al., 2015). The most predominant genus (Table 8), Ruminococcaceae UCG-005 $(P=0.036)$, was affected by RP-Leu and RES levels. The changes in relative abundance of amylolytic genera Bacteroides $(P$ $=0.041)$, Succinivibrio $(P<0.001)$, and Ruminococcaceae $(P=0.046)$ matched the changes at their family level among the different treatment groups, suggesting again that RP-Leu might have decreased the amount of starch fermented in the hindgut.

In summary, the affected blood glucose concentration, changed hindgut fermentation profiles, and altered fecal microbial composition suggest that the effect of RPLeu on small intestinal starch digestion was positively correlated with RES levels and that RP-Leu probably affects starch digestion primarily in the small intestine.

\section{CONCLUSIONS}

In our experiments, RP-Leu supplementation increased blood glucose concentration, decreased fecal excretion of butyrate and propionate, increased relative abundance of fecal cellulolytic bacteria, and decreased relative abundance of amylolytic bacteria. We conclude that RP-Leu probably increased starch digestion in the small intestine while decreasing the flow of starch from the small intestine to the hindgut. The interaction between RP-Leu and RES on fecal butyrate molar proportion and some bacterial genera and families further indicates that RP-Leu affects starch digestion primarily in the small intestine of calves. Overall, our results suggest that RP-Leu could stimulate starch digestion in the small intestine in calves possibly beyond serving as a precursor of protein.

\section{ACKNOWLEDGMENTS}

This work was financially supported by the National Natural Science Foundation of China (award no. 31672451 and 31472122; Beijing). We appreciate the staff numbers of Modern Farm (Baoji, China) for their assistance in animal care. The authors also acknowledge the input of the members of the Innovative Research Team of Animal Nutrition and Health at the Northwest A\&F University (Yangling, Shaanxi, China) during the experimental periods and sample analysis. The authors declare that no conflicts of interest exist.

\section{REFERENCES}

Anderson, J. D., and J. N. Trapp. 2000. The dynamics of feeder cattle market responses to corn price change. J. Agric. Appl. Econ. 32:493-505. https://doi.org/10.1017/S1074070800020599.

Ansia, I., H. H. Stein, M. R. Murphy, and J. K. Drackley. 2019. Technical note: Establishment of an ileal cannulation technique in preweaning calves and use of a piecewise regression approach to evaluate effects on growth and $\mathrm{pH}$ fluctuation of ileal digesta. J. Dairy Sci. 102:11061-11066. https://doi.org/10.3168/jds.2019-16788.

Anthony, J. C., F. Yoshizawa, T. G. Anthony, T. C. Vary, L. S. Jefferson, and S. R. Kimball. 2000. Leucine stimulates translation initiation in skeletal muscle of postabsorptive rats via a rapamycin-sensitive pathway. J. Nutr. 130:2413-2419. https://doi.org/10 .1093/jn/130.10.2413. 
AOAC International. 2002. Official Methods of Analysis. 17th ed. AOAC International, Gaithersburg, MD.

Barham, D., and P. Trinder. 1972. An improved colour reagent for the determination of blood glucose by the oxidase system. Analyst 97:142-145. https://doi.org/10.1039/an9729700142.

Bergman, E. N., D. J. Starr, and S. S. Reulein Jr.. 1968. Glycerol metabolism and gluconeogenesis in the normal and hypoglycemic ketonic sheep. Am. J. Physiol. 215:874-880.

Corona, L., S. Rodriguez, R. A. Ware, and R. A. Zinn. 2005. Comparative effects of whole, ground, dry-rolled, and steam-flaked corn on digestion and growth performance in feedlot cattle. Prof. Anim. Sci. 21:200-206. https://doi.org/10.15232/S1080-7446(15)31203-1.

Doelman, J., J. J. M. Kim, M. Carson, J. A. Metcalf, and J. P. Cant. 2015. Branched-chain amino acid and lysine deficiencies exert different effects on mammary translational regulation. J. Dairy Sci. 98:7846-7855. https://doi.org/10.3168/jds.2015-9819.

Giuberti, G., A. Gallo, F. Masoero, L.F. Ferraretto, P.C. Hoffman, and R.D. Shaver. 2014. Factors affecting starch utilization in large animal food production system: A review. Starch 66:72-90.

Guo, L., Z. Liang, C. Zheng, B. Liu, Q. Yin, Y. Cao, and J. Yao. 2018. Leucine affects $\alpha$-amylase synthesis through PI3K/Akt-mTOR signaling pathways in pancreatic acinar cells of dairy calves. J. Agric. Food Chem. 66:5149-5156. https://doi.org/10.1021/acs.jafc $.8 \mathrm{~b} 01111$

Harmon, D. L. 2009. Understanding starch utilization in the small intestine of cattle. Asian-Australas. J. Anim. Sci. 22:915-922. https: //doi.org/10.5713/ajas.2009.r.08.

Harmon, D. L., R. M. Yamka, and N. A. Elam. 2004. Factors affecting intestinal starch digestion in ruminants: A review. Can. J. Anim. Sci. 84:309-318. https://doi.org/10.4141/A03-077.

Henderson, G., F. Cox, S. Ganesh, A. Jonker, W. Young, Global Rumen Census Collaborators, and P. H. Janssen. 2015. Rumen microbial community composition varies with diet and host, but a core microbiome is found across a wide geographical range. Sci. Rep. 5:14567. https://doi.org/10.1038/srep14567.

Huntington, G. B. 1997. Starch utilization by ruminants: From basics to the bunk. J. Anim. Sci. 75:852-867. https://doi.org/10.2527/ 1997.753852x.

Huntington, G. B., D. L. Harmon, and C. J. Richards. 2006. Sites, rates, and limits of starch digestion and glucose metabolism in growing cattle. J. Anim. Sci. 84(Suppl. 13):E14-E24. https://doi .org/10.2527/2006.8413_supplE14x.

Kotarski, S. F., R. D. Waniska, and K. K. Thurn. 1992. Starch hydrolysis by the ruminal microflora. J. Nutr. 122:178-190. https://doi .org/10.1093/jn/122.1.178.

Kung, L. Jr., and L. M. Rode. 1996. Amino acid metabolism in ruminants. Anim. Feed Sci. Technol. 59:167-172. https://doi.org/10 .1016/0377-8401(95)00897-7.

Lee, S. B., C. W. Choi, Y. C. Jin, T. Wang, K. H. Lee, M. B. Ku, J. H. Hwang, K. H. Kim, R. S. Vega, and H. G. Lee. 2013. Effect of oral administration of intact casein on gastrointestinal hormone secretion and pancreatic $\alpha$-amylase activity in Korean native steer. Asian-Australas. J. Anim. Sci. 26:654-660. https://doi.org/ 10.5713/ajas.2012.12510.

Lesmeister, K. E., and A. J. Heinrichs. 2004. Effects of corn processing on growth characteristics, rumen development, and rumen parameters in neonatal dairy calves. J. Dairy Sci. 87:3439-3450. https:// doi.org/10.3168/jds.S0022-0302(04)73479-7.

Li, F., X. J. Yang, Y. C. Cao, S. X. Li, J. H. Yao, Z. J. Li, and F. F. Sun. 2014. Effects of dietary effective fiber to rumen degradable starch ratios on the risk of sub-acute ruminal acidosis and rumen content fatty acids composition in dairy goat. Anim. Feed Sci. Technol. 189:54-62. https://doi.org/10.1016/j.anifeedsci.2013.12 .011 .

Liao, S. F., E. S. Vanzant, D. L. Harmon, K. R. Mcleod, J. A. Boling, and J. C. Matthews. 2009. Ruminal and abomasal starch hydrolysate infusions selectively decrease the expression of cationic amino acid transporter mRNA by small intestinal epithelia of forage-fed beef steers. J. Dairy Sci. 92:1124-1135. https://doi.org/10.3168/ jds.2008-1521.
Liu, J., M. Zhang, C. Xue, W. Zhu, and S. Mao. 2016. Characterization and comparison of the temporal dynamics of ruminal bacterial microbiota colonizing rice straw and alfalfa hay within ruminants. J. Dairy Sci. 99:9668-9681. https://doi.org/10.3168/jds .2016-11398.

Liu, K., Y. Liu, S. M. Liu, M. Xu, Z. P. Yu, X. Wang, Y. C. Cao, and J. H. Yao. 2015. Relationships between leucine and the pancreatic exocrine function for improving starch digestibility in ruminants. J. Dairy Sci. 98:2576-2582. https://doi.org/10.3168/jds.2014-8404.

Lobley, G. E. 1992. Control of the metabolic fate of amino acids in ruminants: A review. J. Anim. Sci. 70:3264-3275. https://doi.org/ 10.2527/1992.70103264x.

Lomax, M. A., and G. D. Baird. 1983. Blood flow and nutrient exchange across the liver and gut of the dairy cow: Effects of lactation and fasting. Br. J. Nutr. 49:481-496. https://doi.org/10.1079/ BJN19830057.

Luo, G., W. Xu, J. Yang, Y. Li, L. Zhang, Y. Wang, C. Lin, and Y. Zhang. 2017. Effects of ruminally degradable starch levels on performance, nitrogen balance, and nutrient digestibility in dairy cows fed low corn-based starch diets. Asian-Australas. J. Anim. Sci. 30:653-659. https://doi.org/10.5713/ajas.16.0371.

Mao, S., R. Zhang, D. Wang, and W. Zhu. 2012. The diversity of the fecal bacterial community and its relationship with the concentration of volatile fatty acids in the feces during subacute rumen acidosis in dairy cows. BMC Vet. Res. 8:237. https://doi.org/10 $.1186 / 1746-6148-8-237$.

Mao, S. Y., R. Y. Zhang, D. S. Wang, and W. Y. Zhu. 2013. Impact of subacute ruminal acidosis (SARA) adaptation on rumen microbiota in dairy cattle using pyrosequencing. Anaerobe 24:12-19. https://doi.org/10.1016/j.anaerobe.2013.08.003.

Meale, S. J., S. Li, P. Azevedo, H. Derakhshani, J. C. Plaizier, E. Khafipour, and M. A. Steele. 2016. Development of ruminal and fecal microbiomes are affected by weaning but not weaning strategy in dairy calves. Front. Microbiol. 7:582. https://doi.org/10 $.3389 /$ fmicb.2016.00582.

Moharrery, A., M. Larsen, and M. R. Weisbjerg. 2014. Starch digestion in the rumen, small intestine, and hind gut of dairy cows - A meta-analysis. Anim. Feed Sci. Technol. 192:1-14. https://doi.org/ 10.1016/j.anifeedsci.2014.03.001.

Nocek, J. E. 1997. Bovine acidosis: Implications on laminitis. J. Dairy Sci. 80:1005-1028. https://doi.org/10.3168/jds.S0022 -0302(97)76026-0.

Nocek, J. E., and S. Tamminga. 1991. Site of digestion of starch in the gastrointestinal tract of dairy cows and its effect on milk yield and composition. J. Dairy Sci. 74:3598-3629. https://doi.org/10.3168/ jds.S0022-0302(91)78552-4.

NRC. 2001. Nutrient Requirements of Dairy Cattle. 7th rev. ed. Natl. Acad. Press, Washington, DC.

Offner, A., A. Bach, and D. Sauvant. 2003. Quantitative review of in situ starch degradation in the rumen. Anim. Feed Sci. Technol. 106:81-93. https://doi.org/10.1016/S0377-8401(03)00038-5.

Oikonomou, G., A. G. Teixeira, C. Foditsch, M. L. Bicalho, V. S. Machado, and R. C. Bicalho. 2013. Fecal microbial diversity in pre-weaned dairy calves as described by pyrosequencing of metagenomic 16S rDNA. Associations of Faecalibacterium species with health and growth. PLoS One 8:e63157. https://doi.org/10.1371/ journal.pone.0063157.

Ørskov, E. R., C. Fraser, and R. N. Kay. 1969. Dietary factors influencing the digestion of starch in the rumen and small and large intestine of early weaned lambs. Br. J. Nutr. 23:217-226. https:// doi.org/10.1079/BJN19690029.

Ørskov, E. R., C. Fraser, V. C. Mason, and S. O. Mann. 1970. Influence of starch digestion in the large intestine of sheep on caecal fermentation, caecal microflora and faecal nitrogen excretion. Br. J. Nutr. 24:671-682. https://doi.org/10.1079/BJN19700068.

Ørskov, E. R., and N. A. MacLeod. 1990. Dietary-induced thermogenesis and feed evaluation in ruminants. Proc. Nutr. Soc. 49:227-237. https://doi.org/10.1079/PNS19900026.

Ørskov, E. R., and I. McDonald. 1979. The estimation of protein degradability in the rumen from incubation measurements weighted 
according to rate of passage. J. Agric. Sci. 92:499-503. https://doi .org/10.1017/S0021859600063048.

Owens, C. E., R. A. Zinn, A. Hassen, and F. N. Owens. 2016. Mathematical linkage of total-tract digestion of starch and neutral detergent fiber to their fecal concentrations and the effect of site of starch digestion on extent of digestion and energetic efficiency of cattle. Prof. Anim. Sci. 32:531-549. https://doi.org/10.15232/pas .2016-01510.

Owens, F. N., R. A. Zinn, and Y. K. Kim. 1986. Limits to starch digestion in the ruminant small intestine. J. Anim. Sci. 63:1634-1648. https://doi.org/10.2527/jas1986.6351634x.

Rahmatullah, M., and T. R. Boyde. 1980. Improvements in the determination of urea using diacetyl monoxime: Methods with and without deproteinisation. Clin. Chim. Acta 107:3-9. https://doi .org/10.1016/0009-8981(80)90407-6.

Richards, C. J., A. F. Branco, D. W. Bohnert, G. B. Huntington, M. Macari, and D. L. Harmon. 2002. Intestinal starch disappearance increased in steers abomasally infused with starch and protein. J. Anim. Sci. 80:3361-3368. https://doi.org/10.2527/2002 $.80123361 \mathrm{x}$.

Richardson, C. R., and E. E. Hatfield. 1978. The limiting amino acids in growing cattle. J. Anim. Sci. 46:740-745. https://doi.org/10 $.2527 /$ jas1978.463740x.

Robbers, K., E. Westreicher-Kristen, A. Troescher, and A. Susenbeth. 2019. Effects of abomasally infused amylase and increasing amounts of corn starch on fecal excretion of starch, total and microbial nitrogen, and volatile fatty acids in heifers. J. Anim. Sci. 97:3984-3993. https://doi.org/10.1093/jas/skz243.

Rogers, J. A., S. B. Peirce-Sandner, A. M. Papas, C. E. Polan, C. J. Sniffen, T. V. Muscato, C. R. Staples, and J. H. Clark. 1989. Production responses of dairy cows fed various amounts of rumenprotected methionine and lysine. J. Dairy Sci. 72:1800-1817. https: //doi.org/10.3168/jds.S0022-0302(89)79297-3.

Rossi, F., M. Maurizio, M. Francesco, C. Giovanna, and P. Gianfranco. 2003. Rumen degradation and intestinal digestibility of rumen protected amino acids: Comparison between in situ and in vitro data. Anim. Feed Sci. Technol. 108:223-229. https://doi.org/10.1016/ S0377-8401(03)00131-7.

Rulquin, H., and P. M. Pisulewski. 2006. Effects of graded levels of duodenal infusions of leucine on mammary uptake and output in lactating dairy cows. J. Dairy Res. 73:328-339. https://doi.org/10 .1017/S0022029906001841.

Russell, J. B., and J. L. Rychlik. 2001. Factors that alter rumen microbial ecology. Science 292:1119-1122. https://doi.org/10.1126/ science. 1058830.

Sato, T., Y. Ito, T. Nedachi, and T. Nagasawa. 2014. Lysine suppresses protein degradation through autophagic-lysosomal system in C2C12 myotubes. Mol. Cell. Biochem. 391:37-46. https://doi .org/10.1007/s11010-014-1984-8.

Swanson, K. C., J. A. Benson, J. C. Matthews, and D. L. Harmon. 2004. Pancreatic exocrine secretion and plasma concentration of some gastrointestinal hormones in response to abomasal infusion of starch hydrolyzate and/or casein. J. Anim. Sci. 82:1781-1787. https://doi.org/10.2527/2004.8261781x.

Swanson, K. C., J. C. Matthews, C. A. Woods, and D. L. Harmon. 2002. Postruminal administration of partially hydrolyzed starch and casein influences pancreatic alpha-amylase expression in calves. J. Nutr. 132:376-381. https://doi.org/10.1093/jn/132.3 .376 .

Ternouth, J. H., and H. L. Buttle. 1973. Concurrent studies on the flow of digesta in the duodenum and of exocrine pancreatic secretion of calves: The collection of the exocrine pancreatic secretion from a duodenal cannula. Br. J. Nutr. 29:387-397. https://doi .org/10.1079/BJN19730116.

Tiscornia, O. M., M. Singer, J. P. Mendes de Oliveira, P. Demol, and H. Sarles. 1977. Exocrine pancreas response to a test meal in the dog. Am. J. Dig. Dis. 22:769-774. https://doi.org/10.1007/ BF01694506.

Trompette, A., E. S. Gollwitzer, K. Yadava, A. K. Sichelstiel, N. Sprenger, C. Ngom-Bru, C. Blanchard, T. Junt, L. P. Nicod, N. L.
Harris, and B. J. Marsland. 2014. Gut microbiota metabolism of dietary fiber influences allergic airway disease and hematopoiesis. Nat. Med. 20:159-166. https://doi.org/10.1038/nm.3444.

Ulyatt, M. J., D. W. Dellow, C. S. Reid, and T. S. Bauchop. 1975. Structure and function of the large intestine of ruminants. Dig. Metab. Rumin. 77:119-133.

Van Keulen, J. V., and B. A. Young. 1977. Evaluation of acid-insoluble ash as a natural marker in ruminant digestibility studies. J. Anim. Sci. 44:282-287. https://doi.org/10.2527/jas1977.442282x.

Van Soest, P. J., J. B. Robertson, and B. A. Lewis. 1991. Methods for dietary fiber, neutral detergent fiber, and nonstarch polysaccharides in relation to animal nutrition. J. Dairy Sci. 74:3583-3597. https://doi.org/10.3168/jds.S0022-0302(91)78551-2.

Westreicher-Kristen, E., K. Robbers, R. Blank, A. Tröscher, U. Dickhoefer, S. Wolffram, and A. Susenbeth. 2018. Postruminal digestion of starch infused into the abomasum of heifers with or without exogenous amylase administration. J. Anim. Sci. 96:1939-1951. https://doi.org/10.1093/jas/sky082.

Williams, J. E., S. A. Newell, B. W. Hess, and E. Scholljegerdes. 1999. Influence of rumen-protected methionine and lysine on growing cattle fed forage and corn based diets. J. Prod. Agric. 12:696-701. https://doi.org/10.2134/jpa1999.0696.

$\mathrm{Wu}$, G. 2010. Functional amino acids in growth, reproduction, and health. Adv. Nutr. 1:31-37. https://doi.org/10.3945/an.110.1008.

Wu, G. 2013. Functional amino acids in nutrition and health. Amino Acids 45:407-411. https://doi.org/10.1007/s00726-013-1500-6.

Xue, F., Z. Zhou, L. Ren, and Q. Meng. 2011. Influence of rumen-protected lysine supplementation on growth performance and plasma amino acid concentrations in growing cattle offered the maize stalk silage/maize grain-based diet. Anim. Feed Sci. Technol. 169:61-67. https://doi.org/10.1016/j.anifeedsci.2011.05.011.

Yoav, S., Y. Barak, M. Shamshoum, I. Borovok, R. Lamed, B. Dassa, Y. Hadar, E. Morag, and E. A. Bayer. 2017. How does cellulosome composition influence deconstruction of lignocellulosic substrates in Clostridium (Ruminiclostridium) thermocellum DSM 1313? Biotechnol. Biofuels 10:222. https://doi.org/10.1186/s13068-017-0909 $-7$.

Yu, Z. P., M. Xu, K. Liu, J. H. Yao, H. X. Yu, and F. Wang. 2014 Leucine markedly regulates pancreatic exocrine secretion in goats. J. Anim. Physiol. Anim. Nutr. (Berl.) 98:169-177. https://doi.org/ 10.1111/jpn.12069.

Zabielski, R., and S. G. Pierzynowski. 2001. Development and regulation of pancreatic juice secretion in cattle. State-of-the-art. J. Anim. Feed Sci. 10:25-45. https://doi.org/10.22358/jafs/67939/ 2001.

Zhong, R. Z., J. G. Li, Y. X. Gao, Z. L. Tan, and G. P. Ren. 2008. Effects of substitution of different levels of steam-flaked corn for finely ground corn on lactation and digestion in early lactation dairy cows. J. Dairy Sci. 91:3931-3937. https://doi.org/10.3168/ jds.2007-0957.

Zhou, X. Q., Y. D. Zhang, M. Zhao, T. Zhang, D. Zhu, D. P. Bu, and J. Q. Wang. 2015. Effect of dietary energy source and level on nutrient digestibility, rumen microbial protein synthesis, and milk performance in lactating dairy cows. J. Dairy Sci. 98:7209-7217. https://doi.org/10.3168/jds.2015-9312.

Zinn, R. A. 1987. Influence of lasalocid and monensin plus tylosin on comparative feeding value of steam-flaked versus dry-rolled corn in diets for feedlot cattle. J. Anim. Sci. 65:256-266. https://doi.org/ $10.2527 /$ jas1987.651256x.

\section{ORCIDS}

Hao Ren ๑ https://orcid.org/0000-0001-8955-7793

Shengru Wu (1) https://orcid.org/0000-0002-1046-3477

Yangchun Cao @ https://orcid.org/0000-0003-1033-2909

Junhu Yao @ https://orcid.org/0000-0002-0848-8392 